\title{
Incidence of breast cancer and methylation of PCDH gene: a single ratio meta analysis of single rate
}

\section{Type}

Research paper

\section{Keywords}

Breast Cancer, Gene Methylation, PCDH

\begin{abstract}
Introduction

Breast cancer presents one of the highest rates of prevalence. With the development of genetics and biotechnology, we have learned that the occurrence and development of many cancers are closely related to abnormal gene expression. At present, some pieces of literature have reported that there may be a correlation between the expression of PCDH and the occurrence of breast cancer.Therefore, we selected some loci from PCDH gene to explore the correlation between the methylation of PCDH gene and breast cancer.
\end{abstract}

\section{Material and methods}

This research is a systematic review and critical appraisal, make a meta-analysis of prospective and retrospective cohort study. Research was conducted through computer Science, Wanfang and Chinese knowledge network databases PubMed, Embase and Network. In a literature search, seven cohort studies were identified. This 12 statistic is used to quantify heterogeneity. A fixed effect model was used to synthesize the results. Regression tests of linear funnel plot asymmetry were used to estimate potential publication bias.

Results

The methylation rate of PCDH gene in breast cancer with lymph node metastasis was $75 \%$, and that in breast cancer without lymph node metastasis was $70 \%$. The methylation rate of PCDH gene was $75 \%$ in breast cancer group with high expression of the $\mathrm{Ki}-67$ gene and $71 \%$ in breast cancer group with low expression of Ki-67 gene.

\section{Conclusions}

According to previous studies, the positive rate of methylation of PCDH gene in breast cancer tissues is higher than that in adjacent tissues, and there is no obvious statistical difference in the correlation between lymphatic metastasis and Ki-67. 
Incidence of breast cancer and methylation of PCDH gene: a single ratio metaanalysis of single rate

Yu Liu, $\mathrm{MD}^{1}$, Lili Zhong, $\mathrm{PhD}^{2}$, Lixing Wang, $\mathrm{MD}^{1}$, Duohan Zhang, $\mathrm{MD}^{1}$, Tingting Hao, $\mathrm{MD}^{1}$, Xiaoling Xiong, $\mathrm{MD}^{1}$, Chao zhang, $\mathrm{PhD}^{1}$, Yinlong $\mathrm{Zhao,} \mathrm{PhD}^{1 *}$

${ }^{1}$ Department of Nuclear Medicine, The Second Hospital of Jilin University, Changchun 130041, China

${ }^{2}$ Jilin Provincial Key Laboratory on Molecular and Chemical Genentic, The Second Haspital of Jilin University, Changchun 130041, China

*He is a corresponding author.

\section{Corresponding author's postal}

Yinlong Zhao, $\mathrm{PhD}$,

Department of Nuclear Medicine, The Second Hospital of Jilin University, Changchun 130041,

China

Email:yinlong@jlu.edu.cn

Tel:+86-0431-81136750

Fax:+86-0431-81136750

Corresponding E-mails:yinlong@jlu.edu.cn

\section{Other author's postal}

Yu Liu,MD 
Postal address:Department of Nuclear Medicine, The Second Hospital of Jilin University,

Changchun 130041, China

Email:870553361@qq.com

Tel:+86-0431-81136750

Fax:+86-0431-81136750

Lili Zhong,PhD,

Jilin Provincial Key Laboratory on Molecular and Chemical Genentic, The Second Haspital of

Jilin University, Changchun 130041, China

Email:zhong11@jlu.edu.cn

Tel:+86-0431-81136506

Fax:+86-0431-81136506

Lixing Wang, MD

Postal address:Department of Nuclear Medicine, The Second Hospital of Jilin University, Changchun 130041, China

Emai1:906617395@qq.com

Tel:+86-0431-81136750

Fax:+86-0431-81136750

Duohan Zhang,MD

Postal address:Department of Nuclear Medicine, The Second Hospital of Jilin University, Changchun 130041, China

Emai1:820143321@qq.com 
Tel:+86-0431-81136750

Fax:+86-0431-81136750

Xiaoliang Xiong,MD

Postal address:Department of Nuclear Medicine, The Second Hospital of Jilin University,

Changchun 130041, China

Email:xiongxiaoliang@jlu.edu.cn

Tel:+86-0431-81136750

Fax:+86-0431-81136750

Tingting Hao,MD

Postal address:Department of Nuclear Medicine, The Second Hospital of Jilin University,

Changchun 130041, China

Email:httjdey1986@163.com

Tel:+86-0431-81136750

Fax:+86-0431-81136750

Chao Zhnag,PhD

Postal address:Department of Nuclear Medicine, The Second Hospital of Jilin University,

Changchun 130041, China

Email:275882303@qq.com

Tel:+86-0431-81136750

Fax:+86-0431-81136750 


\section{Abstract}

\section{Introduction}

Breast cancer presents one of the highest rates of prevalence. With the development of genetics and biotechnology, we have learned that the occurrence and development of many cancers are closely related to abnormal gene expression. At present, some pieces of literature have reported that there may be a correlation between the expression of $\mathrm{PCDH}$ and the occurrence of breast cancer. Therefore, we selected some loci from PCDH gene to explore the correlation between the methylation of PCDH gene and breast cancer.

\section{Method}

This research is a systematic review and critical appraisal, make a meta-analysis of prospective and retrospective cohort study. Research was conducted through computer Science, Wanfang and Chinese knowledge network databases PubMed, Embase and Network. In a literature search, seven cohort studies were identified. This $\mathrm{I}^{2}$ statistic is used to quantify heterogeneity. A fixed effect model was used to synthesize the results. Regression tests of linear funnel plot asymmetry were used to estimate potential publication bias.

\section{Results}


The methylation rate of PCDH gene in breast cancer with lymph node metastasis was $75 \%$, and that in breast cancer without lymph node metastasis was $70 \%$. The methylation rate of PCDH gene was $75 \%$ in breast cancer group with high expression of the Ki- 67 gene and $71 \%$ in breast cancer group with low expression of Ki-67 gene.

\section{Conclusions}

According to previous studies, the positive rate of methylation of PCDH gene in breast cancer tissues is higher than that in adjacent tissues, and there is no obvious statistical difference in the correlation between lymphatic metastasis and Ki-67.

\section{keywords}

Gene Methylation, PCDH, Breast Cancer

\section{Introduction}

Breast cancer has surpassed lung cancer and become the cancer with the highest incidence of cancer in the world. In 2020, there were 2261419 new cases and 684996 deaths of female breast cancer globally, accounting for $24.5 \%$ and $15.5 \%$ of the total new cases and deaths of female cancer respectively [1]. At present, more and more studies have shown that the abnormal expression of some genes is related to the occurrence and development of tumors, for example, some genes are abnormally expressed or not expressed after methylation, which accelerates the occurrence and development of tumors or the metastasis of tumors. In addition to some proto-oncogenes and tumor suppressor genes that we are familiar with before, there are many 
studies currently exploring the correlation between gene methylation and the occurrence, development and prognosis of diseases. For example, CDX2 methylation predicts the prognosis of lung cancer patients[2], and MGMT promoter methylation is a potential prognosis marker of acute methylation[3]. It has been reported that in the Protocadherin (PCDH) gene family, methylation of PCDH gene will affect many diseases, for example, PCDH10 will affect the progression and pathological types of gastric cancer[4], and PCDH19 will affect the occurrence and outcome of epilepsy[5]. It has been reported that methylation of estrogen receptor promoter gene can lead to benign and malignant changes in breast cells[6].At present, it has been reported that gene methylation is related to the occurrence and development of breast cancer, such as ESR-3 and ERS-1[7].At present, more and more studies have shown that the abnormal expression of some genes is related to the occurrence and development of tumors, for example, some genes are abnormally expressed or not expressed after methylation, which accelerates the occurrence and development of tumors or the metastasis of tumors. In this study, PCDH gene is taken as the breakthrough point[8], $\mathrm{PCDH}$ has been reported in the literature, and it is highly expressed in cell signal transduction and signal pathway quotient of tumor cells[9]. DNA methylation is a form of chemical modification of DNA, which changes the genetic expression without changing the DNA sequence. Under the action of DNA methyltransferase, a methyl group is covalently bonded to cytosine 5' carbon position of $\mathrm{CpG}$ dinucleotide in genome. Studies have shown that DNA methylation can cause changes in chromatin structure, DNA conformation, DNA stability and interaction between DNA and protein, thus controlling gene expression[10]. This paper started from exploring the correlation between methylation of $\mathrm{PCDH}$ gene and breast cancer, and also explored whether there was some correlation between methylation of PCDH gene and lymph node metastasis of breast cancer and expression of Ki-67 gene, which provides more diagnostic evidence in biology for clinical practice[11]. 


\section{Materials and methods}

The literature retrieval strategy

The methylation of PCDH gene in Pubmed, Embase, Web of science, Cochrane Library and related literatures of breast cancer diagnosis were searched by computer in database. At the same time, Google Scholar and other search engines were used for supplementary search, and the references included in the literature were traced back, and gray documents and conference papers were searched manually. The retrieval time was from the establishment of each database to July 23 , 2021. The combination of retrieval strategy and free words was connected with Boolean logic operators and retrieved. English key words: protocadherin, pcdh, cadherins, breast cancer, breast cancer of breast tumor, breast cancer of breast neoplases, Methylation

Inclusion and exclusion criteria

Articles meeting the following criteria were included in meta-analysis: (1) prospective and retrospective cohort study of breast cancer; (2) all patients were pathologically confirmed as malignant breast cancer; (3) at least 20 patients with breast cancer were included in the study; (4) cytological or serological detection of methylation of PCDH gene was selected for these patients; (5) the means and methods of detecting methylation of these patients were described in detail. Exclusion criteria of meta-analysis (1) Exclude all comments, case reports and articles without full text (2) Exclude articles that have not been translated into English or Chinese (3) Exclude studies on benign breast tumors, pathological diagnosis or analysis details after surgery (4) Exclude studies that have not been methylated by PCDH gene (5) Exclude non-clinical experimental 
articles.

The EndNote X9 software is used to eliminate duplicate documents, and the documents are screened based on the titles and abstracts of the documents, and then the potential included documents are read in full.

Data Extraction and Quality Assessment

The following data were extracted from each study: (1) the age range of patients, (2) the types of breast tumors, (3) the number of breast cancers confirmed by pathological biopsy, (4) the number and proportion of methylation of genes detected by methylation histology after diagnosis, (5) the number and proportion of methylation of PCDH genes in serum in some studies, and (6) the staging and grading of tumors in some studies Article Screening and Data Extraction.

The following data were extracted from each study: (1) the age range of patients, (2) the types of breast tumors, (3) the number of breast cancers confirmed by pathological biopsy, (4) the number and proportion of methylation of genes detected by methylation histology after diagnosis, (5) the number and proportion of methylation of PCDH genes in serum in some studies, (6) the staging and grading of tumors in these studiesn order to evaluate the quality of the collected literature, all the included articles were scored by Nos. according to the scoring standard, all the literatures were scored at 7-8. The quality of the included literature was good and met the writing needs of this study（Supplementary Table II）.

Statistical analysis 
Adopt stata 15 The heterogeneity of the included literatures was evaluated, and $\mathrm{I} 2<25 \%$ meant that there was no heterogeneity, so random effect model was used for combined analysis, whereas fixed effect model was used. Subgroup analysis was performed based on tumor size, tumor lymph node metastasis and tumor Ki-67 expression. Because of the lack of control group in one-arm experiment, according to the formula in the study of Chen Yuehong et al. In the formula,

$\mathrm{P}=\mathrm{X} / \mathrm{n}$

$\mathrm{SE}(\mathrm{P})=\sqrt{\mathrm{P}(1-\mathrm{P}) / n}$ $\mathrm{x}$ is the occurrence number of an event, and $\mathrm{n}$ is the total number of observed objects. The used condition $\mathrm{n}$ is large enough, and the incidence $\mathrm{p}$ is not close to 0 and 1 .

\section{Result}

According to the search strategy, 424 articles from PubMed, Cochrane Library and Science Network Database were retrieved. No new articles were found after screening references. Among these articles, 29 were excluded because of duplication and 388 were excluded because they did not meet the inclusion criteria: 386 articles were not related to OSNA breast cancer diagnosis; One paper is non-English literature; 1 non-clinical research literature (Figure 1 is about here).A total of 7 experimental studies [12-18] included specimens of breast cancer patients (tablelis about here), all of whom adopted histological diagnosis methods, and some of them added serological pattern comparison (but this article did not discuss serological gene methylation because of lack of sufficient research quantity). According to the formula and evidence-based medicine of Chen Yuehong et al. According to the formula, we can get $\mathrm{P}<0.05$ and $\mathrm{I} 2>50 \%$. There is no obvious heterogeneity in the meta, so meta-analysis is effective.

PCDH gene methylation and clinical rESponse of breast cancer patients (95\% CI 0.47-0.76), 
standard error es is 0.62 . All of the seven studies included were force-controlled studies, which showed that the methylation rate of genes in breast cancer tissues was higher than that in normal people or adjacent tissues. The comprehensive Stata showed that ES is 0.62(fig2 and fig3 is about here), which also indicated that the methylation rate of $\mathrm{PCDH}$ genes was very high in breast cancer tissues.

Subgroup analysis

1 lymph node metastasis

Methylation forest plot of PCDH gene without lymph node metastasis in breast cancer(fig4 is about here)

Methylation forest plot of PCDH gene without lymph node metastasis in breast cancer(fig5 is about here)

The methylation rate of PCDH gene in breast cancer with lymph node metastasis was $75 \%$, and that in breast cancer without lymph node metastasis was $70 \%$. There is no correlation between methylation of PCDH gene and lymphatic metastasis of breast cancer.

2 The expression of Ki-67[19]

Forest plot of methylation of low-expression PCDH gene in ki-67(fig6 is about here)

Forest plot of methylation of high-expression PCDH gene in ki-67(fig7 is about here) The methylation rate of $\mathrm{PCDH}$ gene was $75 \%$ in breast cancer group with high expression of $\mathrm{Ki}-67$ gene and $71 \%$ in breast cancer group with low expression of $\mathrm{Ki}-67$ gene.There is no correlation between methylation of PCDH gene and Ki-67 gene expression in breast cancer. 


\section{Dicussion}

All the studies are within the confidence interval, and the distribution of all the research points is roughly symmetrical, suggesting that there may be publication bias, however, the results do not suggest obvious publication bias. However, the total data of nine of the seven studies used in this paper are not the same locus of the same PCDH gene, so there is still some heterogeneity. In the direction of the experimental design, the overall thinking of experimental design of each research is the same, but considering the specific operation of experimental design, the specific reagents and instruments, and the problems of manual operation, of course, there are different pathological types of specific experimental tissues, which will lead to certain heterogeneity of the article.

Procadherin $(\mathrm{PCDH})$ is a transmembrane protein and a member of the cadherin superfamily, which plays an important role in cell adhesion and regulation of downstream signaling pathway . At present, studies have shown that PCDH is related to gastric cancer, breast cancer, liver cancer and kidney cancer. Generally, the less PCDH is expressed in water, the worse the differentiation degree of these cancers is, and the greater the recurrence time and probability. $\delta$-PCDHs is often abnormally expressed in human malignant tumors, which may affect the occurrence, progression and prognosis of various malignant tumors by changing cell-cell adhesion and regulating cell signaling pathway. It has been proved that $\delta$-PCDHs plays an inhibitory and carcinogenic role in malignant tumors, and the specific role depends not only on the type of $\delta$-PCDHs, but also on the type of tumor cells. At present, CA153 is the most specific biological test for breast cancer. Therefore, detecting the methylation of PCDH gene as a new biological behavior and better 
applying it in clinical practice should be studied in the future. At the same time, we should pay attention to the correlation and molecular biological mechanism between the expression and deletion of PCDH gene and other tumors.

According to the statistical results, the methylation positive rate of $\mathrm{PCDH}$ gene in patients without lymph node metastasis is $70 \%$, and that with lymph node metastasis is $75 \%$. No obvious statistical difference is found, and no obvious gap can be seen, so it is not enough to prove that the methylation of $\mathrm{PCDH}$ gene has obvious correlation with lymph node metastasis of breast cancer. In the aspect of ki-67 gene expression, the positive rate of gene methylation in ki-67 low expression was $71 \%$, and the proportion of ki- 67 high expression was $75 \%$. There was no correlation between the methylation of PCDH gene and ki-67 gene expression in tumor. To sum up, it is possible that the relationship between methylation of PCDH gene and lymph node metastasis of breast cancer and Ki-67 gene expression in breast cancer has not been found due to insufficient sample size and tumor classification.

In this analysis, due to the limitation of sample size and technology, all the breast cancer-related influencing factors were not included in the experimental study, for example, the correlation between P53 gene expression[20] and PCDH gene methylation, and whether the tumor marker CA153 of breast cancer is related to PCDH gene methylation, which can be used as a supplementary detection method for CA153[21] and provide a better detection method for clinic. At the same time, it can also study breast 。 cancer in the future. To study the different pathological types and methylation of PCDH gene[15, 22], explore the special pathological types of breast cancer, and better help clinicians make preoperative operation plans and better evaluate the prognosis of patients. Can PCDH gene methylation in the clinical work for clinical whether further examination or treatment of a reference standard, at the same time PCDH gene of different location of the specific function should also be a new direction of our further research and 
thinking.

\section{Conclusion}

PCDH gene methylation has a certain correlation with the occurrence of breast cancer, but it

should be noted that normal breast tissue and adjacent tissues also have a certain proportion of

methylation in case-control studies, and have a certain false positive rate.

Reference

1. Parkin DM: Global cancer statistics in the year 2000. The Lancet Oncology 2001, 2(9):533-543.

2. Cui H, Zhang Z, Sun X, Fu L, Zhao X, Zhang M, Wang M: CDX2 methylation may predict the prognosis of patients with lung cancer. Archives of Medical Science 2021.

3. Sobieszkoda D, Czech J, Gablo N, Kopanska M, Tabarkiewicz J, Kolacinska A, Robak T, Zawlik I: MGMT promoter methylation as a potential prognostic marker for acute leukemia. Archives of medical science : AMS 2017, 13(6):1433-1441.

4. Jao TM, Fang WH, Ciou SC, Yu SL, Hung YL, Weng WT, Lin TY, Tsai MH, Yang YC: PCDH10 exerts tumor-suppressor functions through modulation of EGFR/AKT axis 
in colorectal cancer. Cancer letters 2021, 499:290-300.

5. Smith L, Singhal N, El Achkar CM, Truglio G, Rosen Sheidley B, Sullivan J, Poduri A: PCDH19-related epilepsy is associated with a broad neurodevelopmental spectrum. Epilepsia 2018, 59(3):679-689.

6. Gao L, Qi X, Hu K, Zhu R, Xu W, Sun S, Zhang L, Yang X, Hua B, Liu G: Estrogen receptor $\beta$ promoter methylation: a potential indicator of malignant changes in breast cancer. Archives of medical science : AMS 2016, 12(1):129-136.

7. Zhou X, Lei S, Li L, Xu T, Gu W, Ma F, Yang R: [Peripheral blood EMR3 gene methylation level is correlated with breast cancer in Chinese women]. Nan fang yi ke da xue xue bao = Journal of Southern Medical University 2021, 41(10):1456-1463.

8. Jao TM, Tsai MH, Lio HY, Weng WT, Chen CC, Tzeng ST, Chang CY, Lai YC, Yen SJ, $\mathrm{Yu}$ SL et al: Protocadherin 10 suppresses tumorigenesis and metastasis in colorectal cancer and its genetic loss predicts adverse prognosis. International journal of cancer 2014, 135(11):2593-2603.

9. Zhao Y, Yang Y, Trovik J, Sun K, Zhou L, Jiang P, Lau TS, Hoivik EA, Salvesen HB, Sun $\mathrm{H}$ et al: Novel PCDH10-Wnt-MALAT1 regulatory axis in endometrioid endometrial adenocarcinoma. Hong Kong medical journal = Xianggang yi xue za zhi 2019, 25 Suppl 7(5):17-22.

10. Fleischer T, Tekpli X, Mathelier A, Wang S, Nebdal D, Dhakal HP, Sahlberg KK, Schlichting E, Børresen-Dale AL, Borgen $\mathrm{E}$ et al: DNA methylation at enhancers identifies distinct breast cancer lineages. Nature communications 2017, 8(1):1379.

11. Rauscher GH, Kresovich JK, Poulin M, Yan L, Macias V, Mahmoud AM, Al-Alem U, Kajdacsy-Balla A, Wiley EL, Tonetti D et al: Exploring DNA methylation changes in promoter, intragenic, and intergenic regions as early and late events in breast cancer formation. BMC cancer 2015, 15:816.

12. Miyamoto K, Fukutomi T, Akashi-Tanaka S, Hasegawa T, Asahara T, Sugimura T, Ushijima T: Identification of $\mathbf{2 0}$ genes aberrantly methylated in human breast cancers. International journal of cancer 2005, 116(3):407-414.

13. Yu JS, Koujak S, Nagase S, Li CM, Su T, Wang X, Keniry M, Memeo L, Rojtman A, Mansukhani $\mathrm{M}$ et al: PCDH8, the human homolog of PAPC, is a candidate tumor suppressor of breast cancer. Oncogene 2008, 27(34):4657-4665.

14. Shan M, Yin H, Li J, Li X, Wang D, Su Y, Niu M, Zhong Z, Wang J, Zhang X et al: Detection of aberrant methylation of a six-gene panel in serum DNA for diagnosis of breast cancer. Oncotarget 2016, 7(14):18485-18494.

15. Liu W, Wu J, Shi G, Yue X, Liu D, Zhang Q: Aberrant promoter methylation of PCDH10 as a potential diagnostic and prognostic biomarker for patients with breast cancer. Oncology letters 2018, 16(4):4462-4470.

16. Hou S, Shan M, Gao C, Feng X, Yang Y, Zhang R, He Y, Zhang G, Zhang L: PCDHGB7 Increases Chemosensitivity to Carboplatin by Inhibiting HSPA9 via Inducing Apoptosis in Breast Cancer. Disease markers 2019, 2019:6131548.

17. Shan M, Zhang L, Liu Y, Gao C, Kang W, Yang W, He Y, Zhang G: DNA Methylation Profiles and Their Diagnostic Utility in BC. Disease markers 2019, 2019:6328503.

18. Kong DD, Fu RZ, Li L, Wang W, Wang SB: Association between the methylation 
status of PCDH17 and the efficacy of neoadjuvant chemotherapy in triple-negative breast cancer. Oncology letters 2020, 20(2):1649-1656.

19. Chen X, He C, Han D, Zhou M, Wang Q, Tian J, Li L, Xu F, Zhou E, Yang K: The predictive value of Ki-67 before neoadjuvant chemotherapy for breast cancer: a systematic review and meta-analysis. Future oncology (London, England) 2017, 13(9):843-857.

20. Kaur RP, Vasudeva K, Kumar R, Munshi A: Role of p53 Gene in Breast Cancer: Focus on Mutation Spectrum and Therapeutic Strategies. Current pharmaceutical design 2018, 24(30):3566-3575.

21. Song X, Liang B, Wang C, Shi S: Clinical value of color Doppler ultrasound combined with serum CA153, CEA and TSGF detection in the diagnosis of breast cancer. Experimental and therapeutic medicine 2020, 20(2):1822-1828.

22. de Ruijter TC, van der Heide F, Smits KM, Aarts MJ, van Engeland M, Heijnen VCG: Prognostic DNA methylation markers for hormone receptor breast cancer: a systematic review. Breast cancer research : BCR 2020, 22(1):13. 


\begin{tabular}{ccccccc}
\hline $\mathrm{n}$ & year & author & country & PCHD & PCHD positive rate & NOS score \\
\hline 1 & 2020 & DE & Chinese & 17 & 81.40 & 8 \\
2 & 2019 & shan M & Chinese & GB7 & 80.41 & 8 \\
3 & 2018 & wentaoliu & Chinese & 10 & 57.65 & 8 \\
4 & 2016 & yin X & Chinese & 17 & 93.20 & 8 \\
5 & 2016 & mingshen & Chinese & GB7 & 55.60 & 8 \\
6 & 2008 & JS Yu-1 & Chinese & 8 & 28.60 & 8 \\
7 & 2008 & JS Yu-2 & Chinese & 8 & 33.30 & 8 \\
8 & 2005 & gongben-1 & Japan & 10 & 33.33 & 7 \\
9 & 2005 & gongben-2 & Japan & GB6 & 61.90 & 7 \\
\hline
\end{tabular}




\begin{tabular}{|c|c|c|c|c|c|c|c|c|c|}
\hline author & $\begin{array}{l}\text { the case determination } \\
\text { appropriate }\end{array}$ & $\begin{array}{l}\text { Representation of } \\
\text { cases }\end{array}$ & Selection of control & Determination of control & $\begin{array}{l}\text { Comparability of design } \\
\text { control } 2\end{array}$ & $\begin{array}{l}\text { Determination of } \\
\text { exposure factors }\end{array}$ & Same method & No response rate & total \\
\hline $\mathrm{DE}$ & 1 & 1 & 0 & 1 & 2 & 1 & 1 & 1 & 8 \\
\hline shan M & 1 & 1 & 1 & 1 & 2 & 0 & 1 & 1 & 8 \\
\hline wentaoliu & 1 & 1 & 0 & 1 & 2 & 1 & 1 & 1 & 8 \\
\hline yin $X$ & 1 & 1 & 1 & 1 & 2 & 0 & 1 & 1 & 8 \\
\hline mingshen & 1 & 1 & 0 & 1 & 2 & 1 & 1 & 1 & 8 \\
\hline JS Yu-1 & 1 & 1 & 1 & 1 & 2 & 0 & 1 & 1 & 8 \\
\hline gongben-1 & 1 & 1 & 1 & 1 & 0 & 1 & 1 & 1 & 7 \\
\hline
\end{tabular}




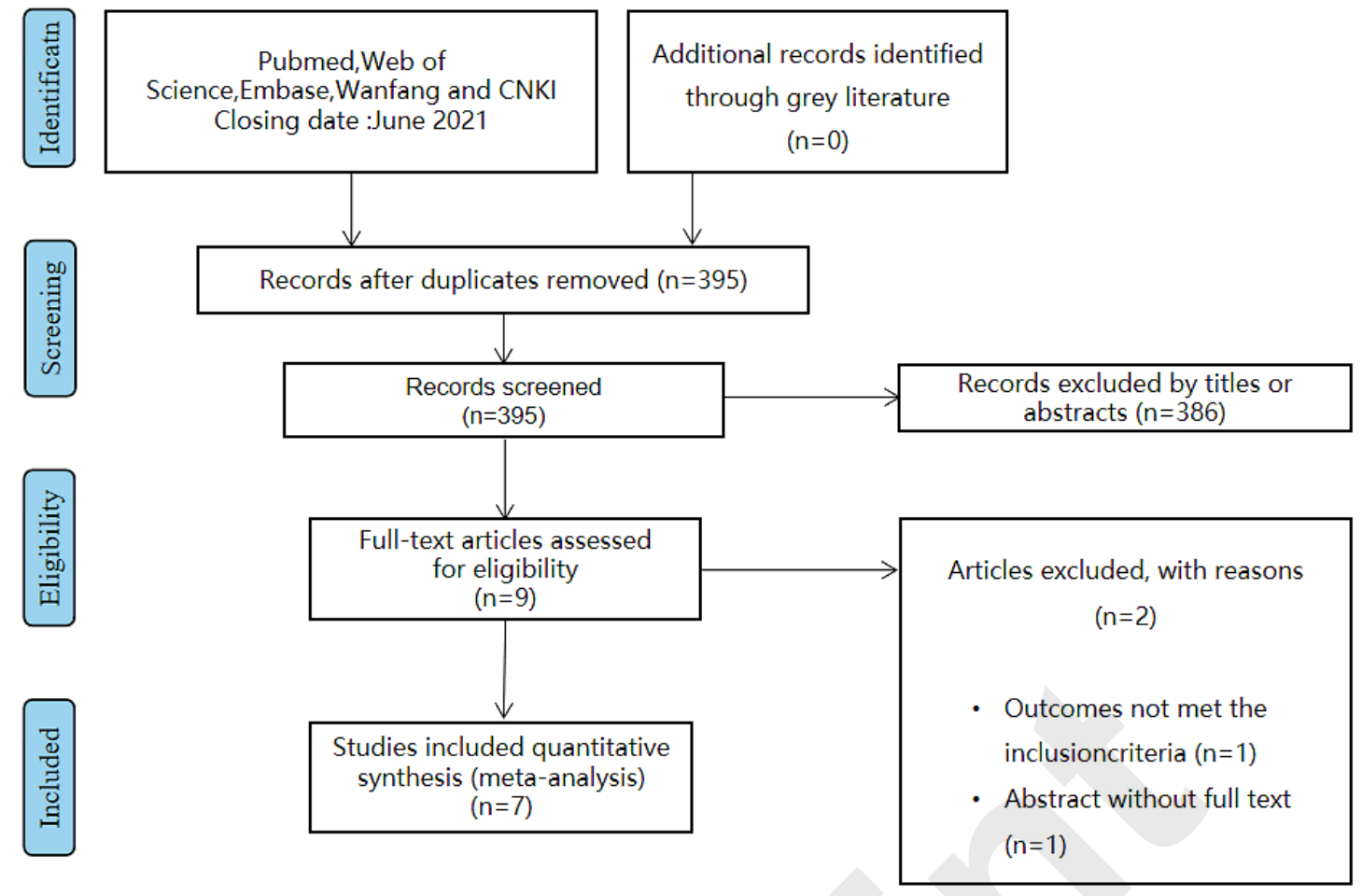




\section{DE (2020)}

shan M (2019)

wentaoliu (2018)

yin $X(2016)$

mingshen (2016)

JS Yu-1 (2008)

JS Yu-2 (2008)

gongben-1 (2005)

gongben-2 (2005)

Overall (I-squared $=2.6 \%, p=0.413$

NOTE: Weights are from random effects analysis

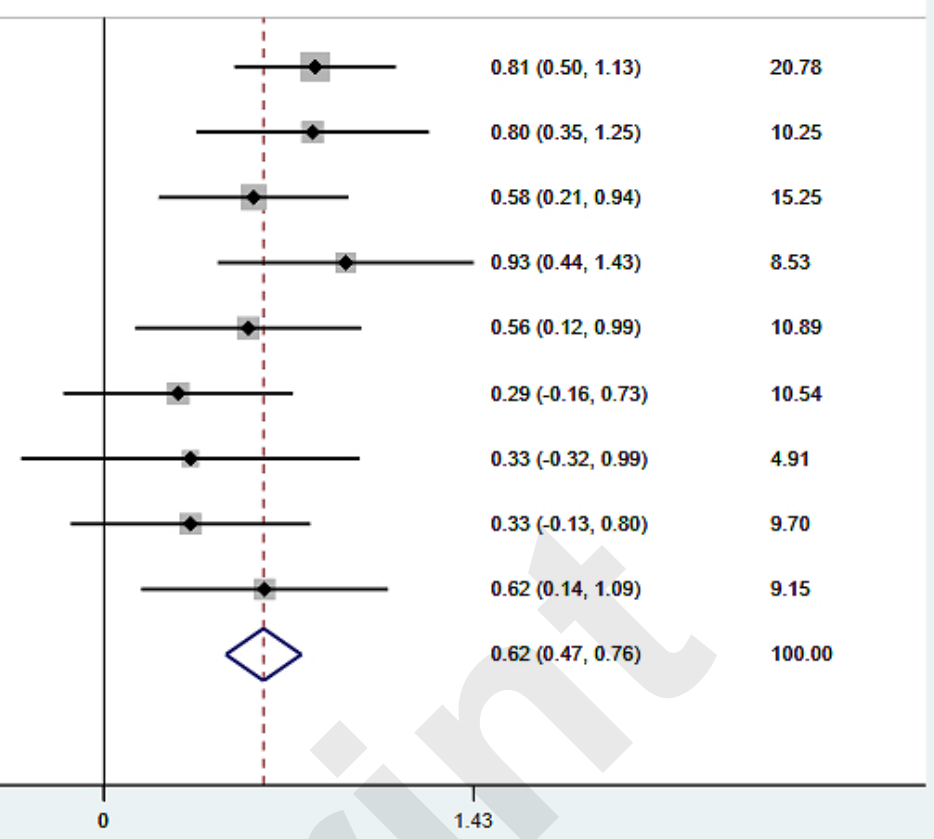


Funnel plot with pseudo $95 \%$ confidence limits

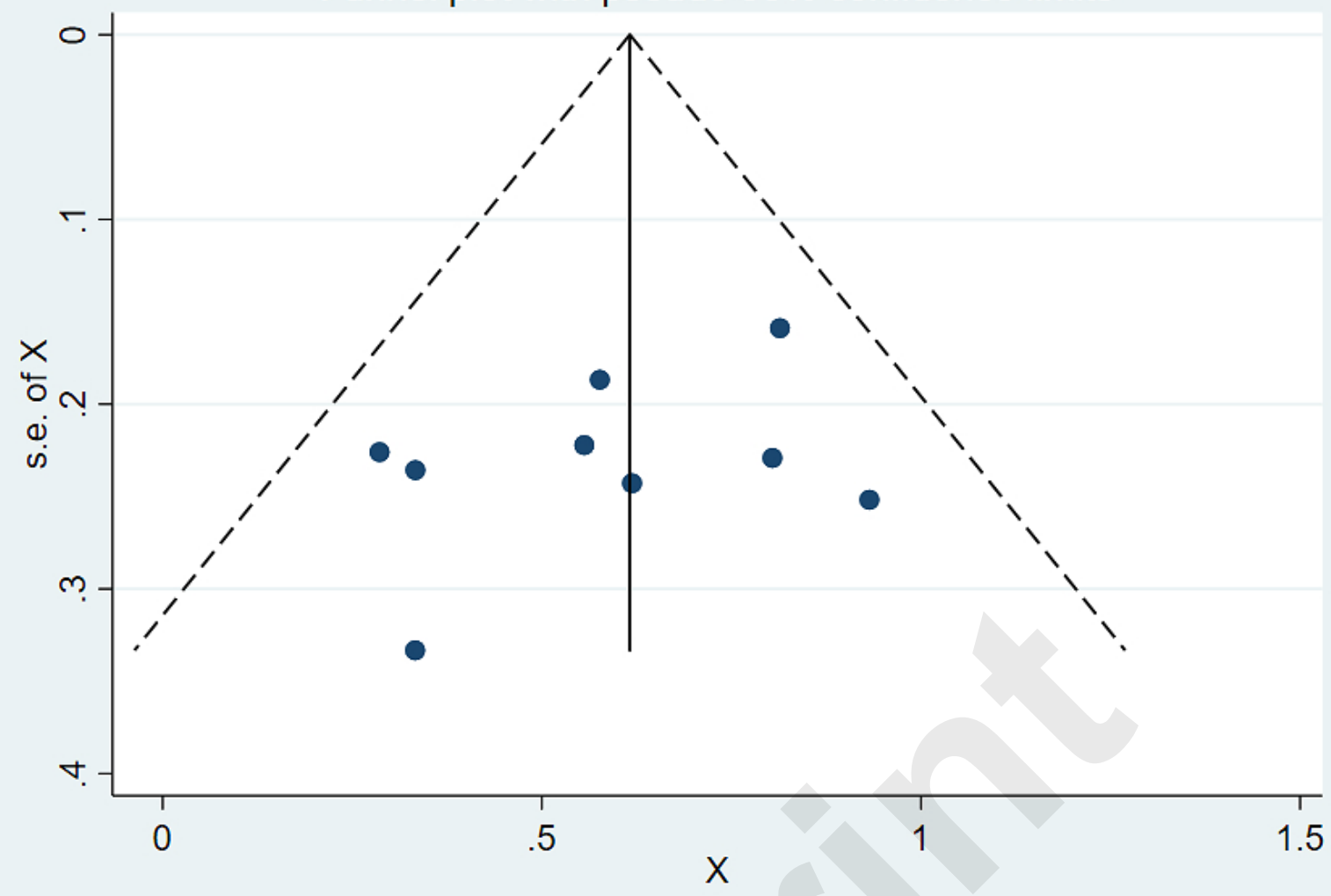


Study

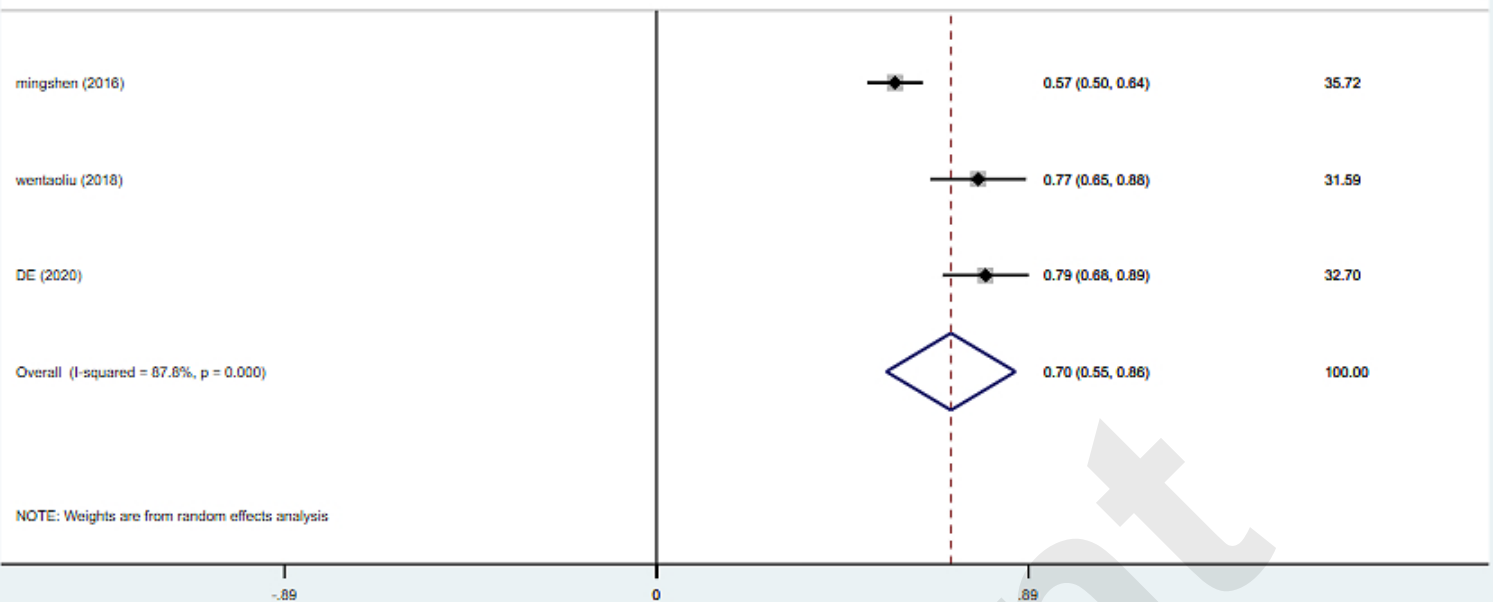

ES (95\% Cl)

Wright 
Study

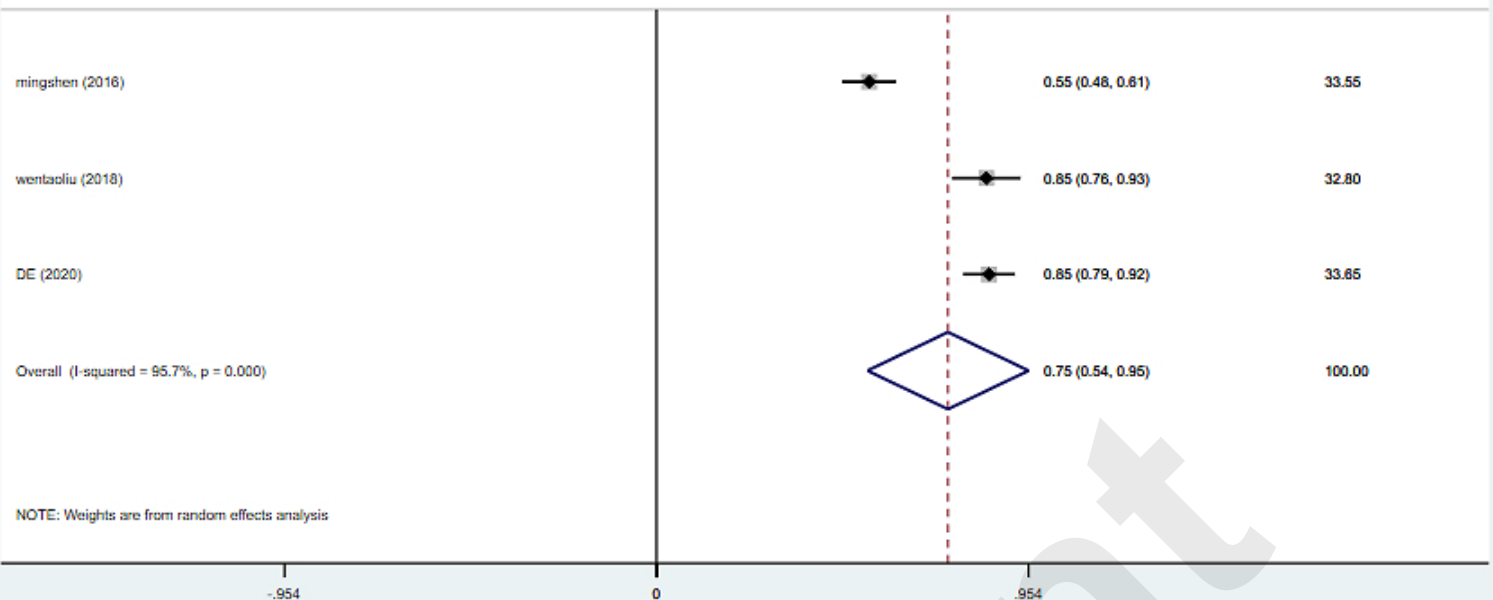

ES (95\% Cl)

Weight 
Study

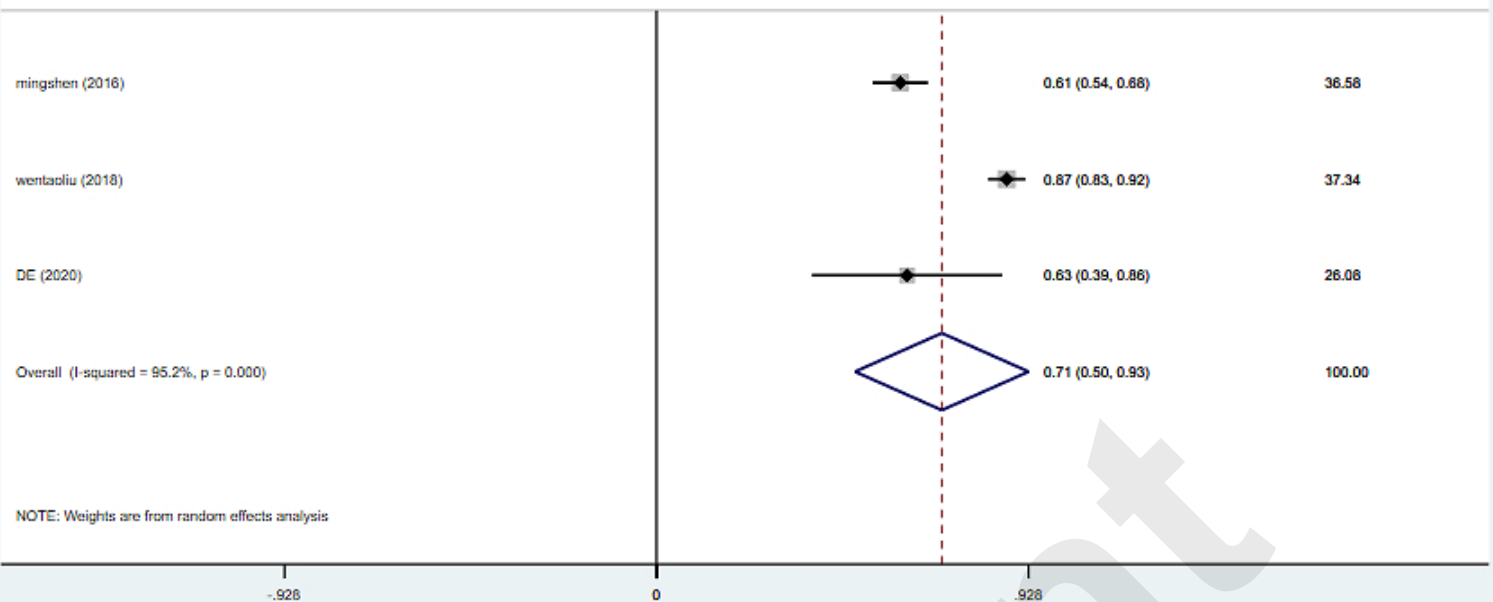

ES ( $95 \%$ Cl)

Wright 
Study

wentacliu (2019)

DE (2020)

Overall (1-squared $=94.2 \%, p=0.000$ )

NOTE: Weights are from randorn effects analyes
$\%$

Weight

33.20

34.39

100.00

ES $(95 \% \mathrm{Cl})$

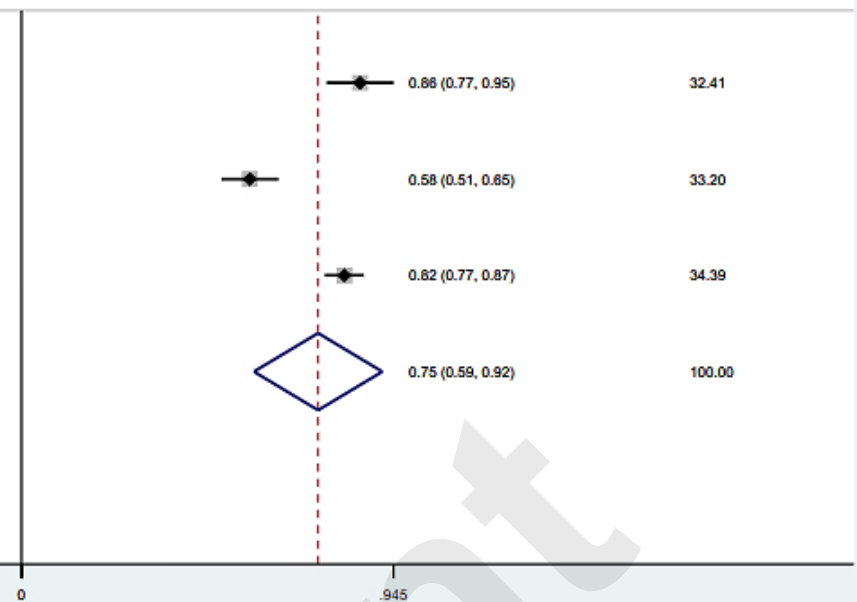

.945 